\title{
Automated three-axis gonioreflectometer for computer graphics applications
}

\author{
Hongsong Li, Sing Choong Foo, Kenneth E. Torrance, and Stephen H. Westin \\ Program of Computer Graphics, Rhodes Hall, Cornell University, Ithaca, NY 14853
}

\begin{abstract}
We describe an automated three-axis BRDF measurement instrument that can help increase the physical realism of computer graphics images by providing light scattering data for the surfaces within a synthetic scene that is to be rendered. To our knowledge, the instrument is unique in combining wide angular coverage (beyond $85^{\circ}$ from the surface normal), dense sampling of the visible wavelength spectrum (1024 samples), and rapid operation (less than ten hours for complete measurement of an isotropic sample). The gonioreflectometer employs a broadband light source and a detector with a diffraction grating and linear diode array. Validation was achieved by comparisons against reference surfaces and other instruments. The accuracy and spectral and angular ranges of the BRDFs are appropriate for computer graphics imagery, while reciprocity and energy conservation are preserved. Measured BRDFs on rough aluminum, metallic silver automotive paint, and a glossy yellow paint are reported, and an example rendered automotive image is included.
\end{abstract}

Keywords: Optics, optical devices, scattering, reflectance, reflectometers, computer graphics

\section{INTRODUCTION}

Realistic rendering is that part of computer graphics that strives to simulate the appearance of three-dimensional scenes in the real world. Accurate physical simulation of light transport and scattering is essential to achieve demonstrably accurate realism, as opposed to a convincing artistic representation. ${ }^{1,2}$ Such simulation differs from most optical simulations in that the output is appearance rather than quantitative data. Further, instead of dealing with a single wavelength and/or a single reflection configuration, we must reproduce the directional and spectral dependence of the reflectance over the entire angular domain and visible spectrum.

The present paper describes a reflectometer to measure the bidirectional reflectance distribution function (BRDF) for computer graphics imagery. The instrument provides

- Broad angular coverage

- High-resolution coverage of the visible spectrum

- Rapid operation

- Accuracy appropriate for computer graphics.

\section{PREVIOUS WORK}

To render surfaces at arbitrary orientations with arbitrary lighting, we wish to maximize the coverage of the incident and reflection hemispheres (with highest grazing angles $>80^{\circ}$ ). To render colors accurately, more than 3 spectral samples are required for each angular configuration. ${ }^{18}$ In order to fully characterize the angular and spectral distributions of the BRDF for a surface, the total number of samples can easily be $10^{4} \sim 10^{5}$. Since we need to characterize many surfaces, the measurement operations have to be reasonably rapid. The limitations of human vision, such as polarization insensitivity and limited dynamic range, can be exploited to accelerate the process. We briefly review previous work.

A classic gonioreflectometer includes a light source, a detector, and some means of varying their angles relative to the sample. ${ }^{3-12}$ A goniometer is often used to hold and rotate the sample so that the angular configuration can be changed repeatedly to cover the incident and reflection hemispheres. Such instruments can achieve broad angular coverage $\mathrm{e}^{3,4}$ and broadband spectral coverage. ${ }^{5,6}$ But none of these instruments combines these capabilities. For example, Germer et al. ${ }^{4}$

Advanced Characterization Techniques for Optics, Semiconductors, and Nanotechnologies II, edited by Angela Duparré, Bhanwar Singh, Zu-Han Gu, Proceedings of SPIE Vol. 5878 (SPIE, Bellingham, WA, 2005)

0277-786X/05/\$15 - doi: 10.1117/12.617589

Proc. of SPIE 58780S-1 
have built an instrument (GOSI) that achieves excellent angular coverage, but limited spectral coverage (3 wavelengths) and long measurement time (weeks for a full angular coverage).

To speed acquisition, multiple detectors can be used in parallel. An example of angular parallelism is using a camera, possibly with special optics, to capture thousands of angles at once, as did Ward et al. ${ }^{13}$ Karner et al. ${ }^{14}$ Castonguay, ${ }^{15}$ Dana et al., ${ }^{16}$ and Marschner et al. ${ }^{17}$ The speed makes such an arrangement popular in the computer graphics community. ${ }^{13,16,17}$ Ward et al. ${ }^{13}$ introduced an image gonioreflectometer that samples two dimensions of the BRDF (two reflection directions) simultaneously, reducing the measurement time to minutes. On the other hand, a camera is generally unable to provide sufficient accuracy for computer graphics, because of its limited spectral coverage (3 channels) and limited dynamic range (8/12 bits).

To measure across a broad range of wavelengths, we could use either a light source or a detector selective to wavelength. The first option can be a broadband source working with a monochromator or a set of narrow band filters. With such an approach, STARR of NIST ${ }^{6}$ and the NASA Goddard scatterometer ${ }^{10}$ provide high-quality BRDF measurements over the UV-Vis-NIR spectrum. But scanning such a broad spectrum takes a long time (hours for STARR). And these instruments usually cover only a portion of the incident and reflection hemispheres. The second option can be a spectroradiometer that measures the entire spectrum at once, ${ }^{5}$ or a narrow-band detector that can vary its wavelength. ${ }^{13}$ Feng et al. ${ }^{5}$ introduced a gonioreflectometer equipped with a spectroradiometer detector, which covers a broad range of spectra in one snapshot. Using a spectroradiometer is faster than the first option, though the dynamic range of the measured signals is generally lower.

None of these instruments displays the combination of angular coverage, wavelength resolution, efficiency, and data quality that we desire. Therefore, we designed and built a gonioreflectometer.

\section{DESCRIPTION OF THE INSTRUMENT}

The gonioreflectometer measures the Bidirectional Reflectance Distribution Function (BRDF), the ratio of the radiance reflected from a surface in the direction $\left(\theta_{r}, \phi_{r}\right)$ to the irradiance onto the surface from the direction $\left(\theta_{i}, \phi_{i}\right)$ (see Figure 1): $f_{r}\left(\theta_{i}, \phi_{i} ; \theta_{r}, \phi_{r}\right)=d L_{r}\left(\theta_{i}, \phi_{i} ; \theta_{r}, \phi_{r}\right) / d E_{i}\left(\theta_{i}, \phi_{i}\right)$. For an isotropic surface only three angles are needed. The reflected radiance $d L_{r}$ and irradiance $d E_{i}$ have units of $\mathrm{W} / \mathrm{m}^{2} \mathrm{sr}$ and $\mathrm{W} / \mathrm{m}^{2}$ respectively.

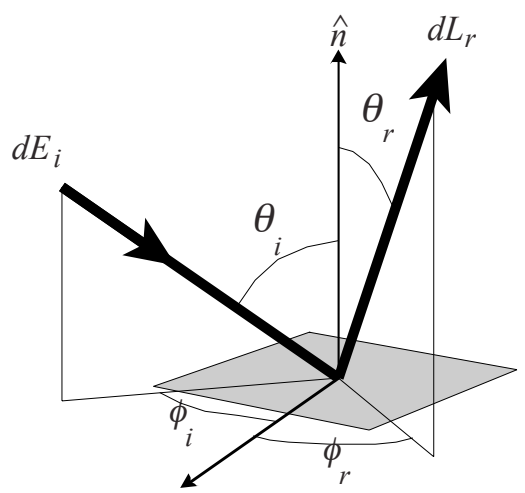

Figure 1. Coordinate system

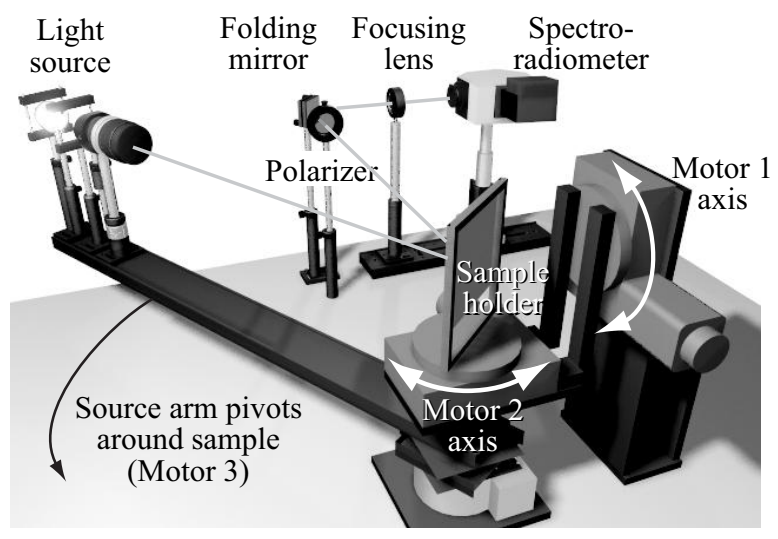

Figure 2. Overview of the Gonioreflectometer

An overview of our gonioreflectometer is shown in Figure 2. The instrument consists of four parts: a broadband light source; a positioning mechanism with three motor-controlled axes of rotation; a fixed spectroradiometer detector; and a computer system (not shown) to control operation, data acquisition, and data processing. The instrument was designed in conformance with the ASTM standard; ${ }^{19}$ instrument parameters are listed in Table 1 . The laboratory is maintained at $20^{\circ} \mathrm{C}$ and a relative humidity of $50 \%$ to aid sample and optical stability. 


\subsection{Light source}

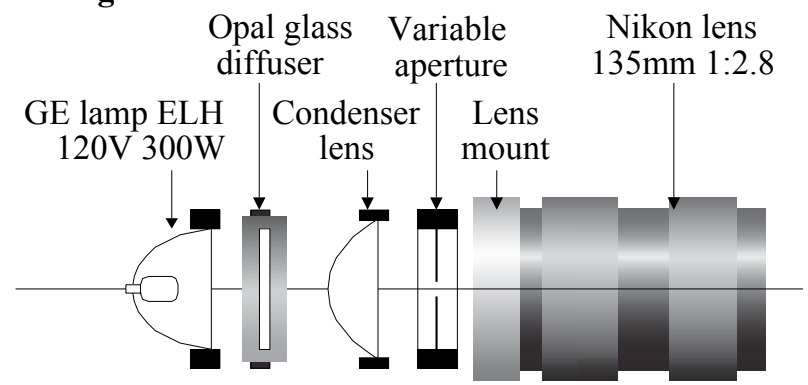

Figure 3. Light source optical components
The light source (Figure 3) is a 300-watt MR16 tungsten halogen lamp with integral dichroic reflector. The source provides a continuous spectrum while minimizing infrared emission, reducing heating of the sample. The beam is depolarized with an opal glass diffuser, gathered by an aspheric condenser lens, passed through a small aperture to approximate a point source, and then collimated by a Nikon camera lens $(f=135 \mathrm{~mm})$ focused at infinity. The source is powered by a regulated, programmable power supply (stable to within $0.03 \%$ to give stable output) and cooled by a fan to avoid deterioration of the dichroic reflector. We

run the lamp at 100 volts rather than its rated 120 volts to prolong its life beyond the rated 35 hours.

The resulting beam is well collimated: it subtends a solid angle of approximately $2.4 \times 10^{-5} \mathrm{sr}$, with illumination uniform to within $\pm 5 \%$ over a circular region of $25 \mathrm{~mm}$ diameter. A scientific grade CCD camera is used to check the uniformity and symmetry of the light spot on the sample surface.

The entire source assembly is mounted on a $1 \mathrm{~m}$ optical rail (Figure 2), which is in turn attached to a motorized rotation stage. A baffled, ventilated housing around the entire source assembly controls stray light. When needed, we mount a dichroic polarizer on the optical rail to control the polarization state of the source; in normal operation we use a depolarized light source.

\subsection{Positioning mechanism}

The sample holder (Figure 2) is mounted on two motor-driven stages to provide two axes of rotation; a third stage, mentioned above, supplies a third axis by moving the source arm in a horizontal plane (parallel to the table). The three axes of rotation provide the three rotational degrees of freedom needed to sample an isotropic BRDF. The configuration is similar to that described by Erb and Krystek. ${ }^{12}$ All three axes are controlled by stepping motors under computer control. All rotational axes intersect at the center of the sample plane, so that measurements at all angles are centered on the same surface point. The sample holder precisely locates the sample plane by means of four supporting lugs, with elastic bands holding the sample with slight pressure from behind. The angular resolution is $0.1^{\circ}$ for the sample stage and $0.13^{\circ}$ for the source arm. The maximum angular error is $0.34^{\circ}$, mainly due to lash in the gear trains of the stages. In most cases, the error is within $0.1^{\circ}$. For BRDF measurements of smooth surfaces, special procedures are used to reduce the error. All surfaces of the sample holder are painted flat black to minimize stray light scatter.

\subsection{Detector}

The detector is designed to accept a broadband signal from the sample, measuring many wavelengths at once for faster operation. The detector unit consists of a folding mirror, focusing optics, and a spectroradiometer (see Figure 2). The position of the entire unit is carefully aligned and fixed. The light scattered by the sample surface is redirected by the folding mirror and focused on the entrance slit of the spectroradiometer through an achromatic doublet. The diffraction grating in the radiometer makes it sensitive to polarization. For this reason, we measure the BRDF twice with different detector polarizations, using a dichroic polarizer in front of the folding mirror to select a single linear polarization for measurement. By averaging the two measurements, and using a detector-polarization calibration curve, we obtain the polarization-averaged reflectance.

The spectroradiometer contains a spectrograph with a reflective interference grating of 600 lines/mm and a 1024-pixel diode array detector, covering the visible spectrum $(386 \mathrm{~nm}-711 \mathrm{~nm})$. We decimate the resolution to 31 output samples (400nm-700nm with 10nm intervals), using a Gaussian kernel, to reduce noise in the output data.

The diode array is thermoelectrically cooled to stabilize its output. The signal resolution is 16 bits. For a fixed exposure time, the dynamic range of the detector is approximately 1:20,000. We verified that the response of the detector is linear over the range that we operate the detector, except at very low signal levels (a few counts). Other experiments confirmed the stability and repeatability of the detector. 
The detector optics determine the viewed sample surface area, which is defined by the magnified image of the detector slit on the sample. At $\theta_{r}=0^{\circ}$ the viewed area is $2 \mathrm{~mm} \times 4 \mathrm{~mm}$; the $2 \mathrm{~mm}$ dimension increases as $\left(\cos \theta_{r}\right)^{-1}$. The minimum diameter of the illuminated spot at normal incidence is $25 \mathrm{~mm}$, so the constraint on $\theta_{r}$ becomes $\theta_{r, \max }=\cos ^{-1}(2 / 25) \sim 85^{\circ}$. The latter represents the fundamental limit on the angular coverage of the instrument: when the projection of the slit exceeds the illuminated spot size, the BRDF will be underestimated. The angular limit compares favorably with other computer graphics instruments such as that of Ward et al., which was limited to approximately $60^{\circ}$ from the normal. ${ }^{22}$

A typical exposure time is 10 seconds at each angular configuration, resulting in measurement times of roughly 9-10 hours for a reasonably diffuse sample (using about $10^{3}$ angular configurations). The control software automatically senses saturation of the detector and reduces exposure time to obtain useful data in the brightest regions of the BRDF, notably near specular peaks.

\subsection{Control software}

The gonioreflectometer is controlled by a $\mathrm{C}$ program that accepts an input file of angular configurations. The file specifies the position of each of the three motors. The program powers up the light source in a controlled, repeatable fashion, then leads the operator through the steps of aligning the three drive motors, measuring detector dark current and the source intensity, and making two sets of measurements, one for each polarization direction. The operator intervenes only at the start to mount the sample, and midway to change the polarizer orientation.

The input file of motor positions is generated offline. For a normal measurement, we generate sampling positions on a regular grid on the unit square. These are then mapped to the unit sphere with a transformation that produces a uniform distribution in solid angle. ${ }^{23}$ The transformation from this space to the coordinates of the three stepper motors is reasonably straightforward, and is developed both by $\mathrm{Foo}^{20}$ and by Erb and Krystek. ${ }^{12}$ Some sampling positions (within approximately $7^{\circ}$ of retro-reflection) are not achievable, due to mechanical interference between the light source and detector. We omit such positions from the command stream sent to the controller, and depend on later interpolation to fill gaps as needed. Further details of the instrument and operating procedures are available in technical reports. ${ }^{20,21}$

\section{CALIBRATIONS AND NORMALIZATION}

\subsection{Absolute method - Spectralon test}

The instrument allows both absolute and relative BRDF measurements, using the ASTM standard protocols. ${ }^{19}$ The absolute method works directly from the ratio of reflected and incident radiant beams. To do this, we first position the source to illuminate the detector directly, capturing the entire source irradiating flux that impinges on the sample. We then measure the reflected flux from the sample at each angular configuration. The spectroradiometric signal for each angular configuration is divided by the signal of the direct light source measurement, the incident cosine, and the solid angle of the detector. The last factor effectively becomes the scale factor.

To verify the directional and magnitude accuracy of our instrument, we compare in Figure 4 our polarized BRDF measurements of Spectralon in the incidence plane with data published by Labsphere. ${ }^{24}$ The incident light was $p$ polarized by installing a horizontal polarizer at the light source. The reflected light of both $s$ and $p$ polarizations was then measured, at a wavelength of $633 \mathrm{~nm}$. The incidence angle was $30^{\circ}$ and the reflection angle varied from $-86^{\circ}$ to $86^{\circ}$. Our $p s$ measurements (the depolarized component) are essentially identical with the published results; the $p p$ measurements (which include surface scatter) differ slightly (by $0.02 \mathrm{sr}^{-1}$ ). We were unable to verify that the surface roughness of our sample matched that of the sample used in the published data, so differences in surface finish between our sample and the sample measured by Labsphere may account for the differences in the two sets of $p s$ measurements.

The absolute normalization method can give good directional and magnitude accuracy for surfaces with strongly specular, or mirror-like, reflection behavior. For surfaces with strongly-diffusing reflection behavior, or with very low diffuse reflectance values (dark surfaces), the alternate relative method is preferred.

\subsection{Relative method}

The relative method of BRDF measurement works by simply comparing the reflected signal from a test sample to that from a reference surface with a known BRDF. To do this, the sample is mounted on the goniometer and the reflected signal is measured for each angular configuration. Subsequently, we measure the reflected signal from the reference sample (Spectralon) at one particular angular configuration, $\theta_{i}=0^{\circ}$ and $\theta_{r}=10^{\circ}(0 / 10)$. The spectroradiometric signal 
from the test sample for each angular configuration is then divided by the signal from the Spectralon at $0 / 10$ and a cosine factor, and multiplied by the absolute BRDF of the Spectralon at 0/10 to give the sample's BRDF.

The absolute BRDF of Spectralon at 0/10 is derived from measurements of both the angular distribution of the reflected signal by using the gonioreflectometer, and the directional-hemispherical reflectance, the latter measured with an Optronic Labs OL-750 diffuse reflectometer. The directional-hemispherical reflectance is the ratio of the radiant power reflected to the hemisphere to the radiant power incident onto a surface, both in $\mathrm{W} / \mathrm{m}^{2}$. Thus, the absolute BRDF of Spectralon at $0 / 10$ becomes the instrument calibration factor.

Our relative method is most similar to the Relative Total Reflectance Method recommended in the ASTM standard. ${ }^{19}$ That method integrates the measured relative BRDFs over the reflection hemisphere and adjusts calibration factors to match a separately-measured directional-hemispherical reflectance. We use a directional-hemispherical reflectance at only one incidence angle $\left(10^{\circ}\right)$ to obtain the calibration factor. This simplification is valid when the reflectance of the test sample is similar to that of the reference sample in magnitude and directionality. In the next two sections, results obtained using the relative method are presented for three samples.

\section{VALIDATION}

Physical laws constrain the BRDF to satisfy two relations that can be checked easily:

- Helmholtz reciprocity requires that $f_{r}\left(\theta_{i}, \phi_{i} ; \theta_{r}, \phi_{r}\right)=f_{r}\left(\theta_{r}, \phi_{r} ; \theta_{i}, \phi_{i}\right)$, and

- energy conservation limits the integral of all energy scattered from the surface:

$\int_{\Omega_{r}} f_{r}\left(\theta_{i}, \phi_{i} ; \theta_{r}, \phi_{r}\right) \cos \theta_{r} d \Omega_{r} \leq 1$

where the integral is over the reflection hemisphere $\Omega_{r}$.

The left side of this inequality is defined as the directional-hemispherical reflectance $\rho_{d h}$. Instruments are commercially available to measure $\rho_{d h}$, giving an opportunity to verify BRDF measurements by numerical integration. In this section, we use a rough aluminum surface to demonstrate the relative method and verify the energy conservation of measurements for a non-trivial surface. We tested reciprocity with the Spectralon sample. Similar verification procedures are applied to the other samples and the results are presented in the next section.

\subsection{Energy conservation - rough aluminum surface}

We tested energy conservation by measuring a rough aluminum surface, which was prepared by coating ground glass with pure aluminum. The resulting RMS roughness was $0.63 \mu \mathrm{m}$.

Figure 5 shows BRDF measurements in the plane of incidence for various illumination angles $\theta_{i}$, using linear interpolation between the dense sample points. The measurements are consistent with measurements by Torrance and Sparrow. ${ }^{25}$ Off-specular peaks beyond the mirror angle of reflection $\left(\theta_{r}=\theta_{i}\right)$ are evident, as is a uniform diffuse (Lambertian) component (to the left in the graph). The off-specular peak increases with increasing incidence angle.

A second set of measurements covered the whole incident and reflection hemispheres. For an incident direction of $10^{\circ}$ and a wavelength of 550nm, the BRDF in the reflection hemisphere is shown in Figure 6. The vertical axis is the BRDF; the left and right orthogonal axes map the spherical coordinates above a surface. The plane of incidence corresponds to 0 on the left axis. The sampling positions were uniformly distributed to capture the basically diffuse character of this surface. The points are plotted in a uniform parameterization of the hemisphere such that each grid in the plot represents a region of the hemisphere with the same solid angle. The mapping is responsible for the sharp-edged artifacts along the diagonals; they do not exist in the data.

We tested energy conservation of these measurements by comparing with another instrument. The BRDF data were integrated over the reflection hemisphere to obtain the directional-hemispherical reflectance. The same sample was also measured with the Optronics OL-750 diffuse reflectometer system, which obtains the directional-hemispherical reflectance of a flat surface at an incidence angle of $10^{\circ}$ with a rated error of less than $1 \%$. Figure 7 shows a comparison of the integrated and direct measurements. The directional-hemispherical reflectance obtained from the gonioreflectometer BRDF measurements matches the direct measurements from the OL-750 within $2 \%$ over the entire visible spectrum, except for the shortest wavelengths, below $420 \mathrm{~nm}$. At these wavelengths, several factors contribute to increased error: the tungsten source of the gonioreflectometer has relatively low output, the silicon-based detector has 
reduced sensitivity, and the dichroic polarizer is much less effective. The comparison suggests indirectly that the BRDF measurements are accurate in both magnitude and spectrum, with less than $2 \%$ integrated error.

\subsection{Reciprocity - Spectralon surface}

The BRDF satisfies reciprocity: when the positions of the light source and detector are interchanged, the measured results should be the same. To verify reciprocity, the light reflection of Spectralon was measured in the plane of incidence, where $\phi_{i}=\phi_{r}=0$. We compared the signal readings of two measurements, each satisfying $\theta_{i 1}=\theta_{r 2}$ and $\theta_{i 2}=\theta_{r 1}$. Representative data, in Table 2, show that reciprocity is satisfied to within $1 \%$ to angles as great as $80^{\circ}$ from the surface normal.

\section{MEASUREMENTS}

In this section, we present measured BRDFs for two additional materials: a metallic silver automotive paint and a glossy yellow paint. In contrast with Section 5, these surfaces represent materials that are more common in typical real-world environments. The silver paint demonstrates the capture of an interesting directional dependence of the BRDF. The silver paint is basically gray in color, as were the materials presented in the previous section. The yellow paint shows our instrument's ability to capture the spectral dependence of the BRDF, resulting in brilliant color.

\subsection{Metallic silver paint}

The metallic silver paint (duPont) scatters light through a more complex mechanism than the rough aluminum surface considered in section 5.1 above: there is an ideal specular reflection from the smooth, glossy surface of the paint, but most light penetrates the surface and is scattered more diffusely from flakes of metal beneath the surface. Figure 8 shows the measured BRDFs in the plane of incidence. The graph is taken from the full hemispherical data set, so shows coarser sampling than that used for Figure 5. We interpolated the sparser data points with a cubic spline after deleting samples near the specular (mirror) direction to better isolate the subsurface scattering from the first-surface reflection. The deleted mirror peak essentially follows the Fresnel formula for mirror reflection from a smooth dielectric surface. In BRDF coordinates, the mirror peaks would be off scale in Figure 8, and would be centered on the mirror reflection angle with a half angle of about $0.4^{\circ}$ due to the small solid angle of the light source.

The paint sample shows a reflectance pattern in Figure 8 that is very different from the rough aluminum surface (Figure 5). First, there is almost no Lambertian component to the left in the figure. Second, the directional lobe is narrower and is centered on the mirror direction (except for $\theta_{i}=80^{\circ}$ ). Third, the increase in BRDF magnitude with increasing $\theta_{i}$ is much smaller, and reverses as $\theta_{i}$ exceeds $70^{\circ}$. We suspect the third effect is due to two factors: the unknown angular distribution of the subsurface metal flakes, and the first-surface reflection (not shown) which tends to divert a larger proportion of the incident light near grazing incidence.

Figure 9 provides an example BRDF for the paint over the mapped reflection hemisphere, for an incident direction of $10^{\circ}$ and a wavelength of 550nm. Figure 10 displays a comparison of the integrated and separately-measured directionalhemispherical reflectances for the paint, over the visible wavelength spectrum. For this figure, the Fresnel component has been excluded from both curves. Figures 9 and 10 respectively resemble their counterparts for the rough aluminum surface, Figures 6 and 7.

\subsection{Glossy yellow paint}

The glossy yellow paint (Krylon 7221 Canary) scatters light from its surface and from subsurface paint pigments. Reflection from the nearly smooth surface is gloss-like, with Fresnel-like reflection at large angles of incidence. The subsurface scattering is wavelength dependent, giving the material its yellow color, and is diffuse in character.

Figure 11displays the measured incidence-plane BRDF for three angles of incidence and two wavelengths. The full hemispherical BRDF data set has been interpolated to get the incidence-plane curves. The Fresnel-like mirror component from the surface has been deleted; that component follows the Fresnel formula for mirror reflection from a smooth dielectric and is nearly independent of wavelength since the index of refraction of the paint binder is nearly independent of wavelength. The peaks appearing near the mirror reflection angles of $10^{\circ}, 40^{\circ}$ and $60^{\circ}$ are due to a glosslike component of surface reflection. The gloss peaks vary slightly in magnitude with wavelength. Away from the peaks, there is a nearly constant (i.e., ideal diffuse) BRDF, which arises from the subsurface reflection. Since the subsurface reflection is wavelength dependent, the diffuse component is wavelength dependent. 
We observe striking differences between the incidence-plane BRDFs for the aluminized ground glass, the metallic paint, and the yellow paint in Figures 5, 8, and 11, respectively. The reflection pattern of the aluminum surface (Figure 5) is due to first surface reflection from the rough surface, accounting for the reflection peaks, and multiple reflections among roughness elements on the surface, producing a nearly constant diffuse pattern. At larger angles of incidence, the BRDF peaks are at reflection angles beyond the mirror reflection angle. The two paints, however, show strong gloss-like peaks near the mirror reflection angle. For the metallic paint (Figure 8), the peaks arise from metal flakes within the paint binder, whereas for the yellow paint (Figure 11), the peaks arise from a surface glossiness. Further, the metallic paint shows only a weak or nonexistent diffuse reflection away from the gloss-like peaks (Figure 8), whereas the yellow paint shows a strong nearly ideal-diffuse reflection away from the glossy peaks (Figure 11).

Figure 12 provides a comparison of the integrated and separately-measured directional-hemispherical reflectances for the yellow paint, over the visible wavelength range, and the agreement is to within a few percent. For this figure, the Fresnel component has been excluded from both curves.

\section{EXAMPLE IMAGE}

Finally, we demonstrate the use of our instrument for its designed purpose by rendering a realistic image of a 3dimensional scene. We adapted the BRDF measurements of silver paint presented in Section 6.1 for rendering by approximating them with smooth basis functions. ${ }^{26}$ This representation offers both proven accuracy and the computational efficiency needed for rendering, where each BRDF may be evaluated millions of times to generate a single image. Figure 13 was then rendered using Blue Moon Rendering Tools, a RenderMan ${ }^{\mathrm{TM}}$-based program that implements high-quality ray tracing. ${ }^{27}$ A custom RenderMan ${ }^{\mathrm{TM}}$ shader program (available at

http://www.graphics.cornell.edu/ westin/lafortune/lafortune.html) was written to implement our BRDF approximation. The car body uses our measured reflectance, with an additional Fresnel term to model reflection from the smooth surface. The convincing appearance of this image demonstrates the usefulness of rendering from actual physical measurements, and the capability of our instrument to provide these measurements.

\section{CONCLUSION}

We have presented an automated three-axis gonioreflectometer designed to meet the needs of computer graphics. The instrument can cover almost the entire angular domain of an isotropic BRDF, covers the entire visible spectrum with ample wavelength resolution, and is rapid enough to measure real materials in a practical time (less than ten hours.) The angular range covers the entire incident and reflection hemispheres to an angle of at least $85^{\circ}$, with the exception of a cone of approximately $7^{\circ}$ around retro-reflection. We measure 31 wavelength samples covering the visible spectrum $(400 \mathrm{~nm}-700 \mathrm{~nm})$ in one snapshot. Our initial measurements have validated the accuracy of the instrument. Computergenerated images based on the measurements are realistic and physically plausible.

\section{ACKNOWLEDGEMENTS}

The authors would like to thank Donald Greenberg, Hurf Sheldon, and Eric P. F. Lafortune of the Cornell Program of Computer Graphics for their encouragement and technical support. Equipment was donated by the Imaging Science Division of Eastman Kodak (Larry Iwan) and the Hewlett-Packard Company; the silver paint sample was donated by Ford Motor Company. This research was supported by the NSF Science and Technology Center for Computer Graphics and Scientific Visualization (ASC-8920219), by the NSF Thermal Systems Program (CTS-9213183), and by the NSF Information Technology Research Program (ACI-0113851). 


\section{REFERENCES}

1. D. P. Greenberg, K. E. Torrance, F. X. Sillion, J. Arvo, J. A. Ferwerda, S. Patanaik, E. P. F. Lafortune, B. Walter, S. C. Foo, and B. Trumbore, "A framework for realistic image synthesis," Comput. Graph. Proc., Annual Conference Series (SIGGRAPH95), 477-494 (1995).

2. X. D. He, K. E. Torrance, F. X. Sillion, and D. P. Greenberg, "A comprehensive physical model for light reflection," Comput. Graph. 25 (SIGGRAPH91), 175-186, 1991.

3. L. Davis and J. G. Kepros, "Improved facility for BRDF/BTDF optical scatter measurements," in Stray Radiation V, R. P. Breault, ed., Proc. SPIE 675, 24-32 (1986).

4. T. A. Germer and C. C. Asmail, "A goniometric optical scatter instrument for bi-directional reflectance distribution function measurements with out-of-plane and polarimetry capabilities," in Scattering and Surface Roughness, A. A. M. Zu-Hun Gu, ed., Proc. SPIE 3141, 220-231 (1997).

5. X. Feng, J. R. Schott, and T. Gallagher, "Comparison of methods for generation of absolute reflectance-factor alues for bi-directional reflectance-distribution function studies," Appl. Opt. 32, 1234-1242, 1993.

6. J. E. Proctor and P. V. Barnes, "NIST high accuracy reference reflectometer-spectrophotometer," J. Res. Natl. Inst. Stand. Technol. 101, 619-627 (1996).

7. J. J. Hsia and J. C. Richmond, "Bidirectional Reflectometry Part I. A high resolution laser bi-directional reflectometer with results on several optical coatings," J. Res. Natl. Inst. Stand. Technol. 80A, 189-205 (1976).

8. S. Mainguy, M. Olivier, M. Josse, and M. Guidon, "Description and calibration of a fully automated infrared scatterometer," in Optical Scatter: Application, Measurement, and Theory, J. C. Stover, ed., Proc. SPIE 1530, 269282 (1991).

9. B. T. McGuckin, D. A. Haner, R. T. Menzies, C. Esproles, and A. M. Brothers, "Directional reflectance characterization facility and measurement methodology," Appl. Opt. 35, 4827-4834, 1996.

10. T. F. Schiff, M. W. Knighton, D. J. Wilson, F. M. Cady, J. C. Stover, and J. J. Butler, "Design review of a highaccuracy UV to near-IR scatterometer," in Optical Scattering: Application, Measurement, and Theory II, J. C. Stover, ed., Proc. SPIE 1995, 121-130 (1993).

11. D. R. White, P. Saunders, S. J. Bonsey, J. van de Ven, and H. Edgar, "Reflectometer for measuring the bidirectional reflectance of rough surfaces," Appl. Opt. 37, 3450-3454, 1998.

12. W. Erb and M. Krystek, "Ein neuartiges 3D-Gonioreflektometer," Optik, 83, 51-54, 1989

13. G. J. Ward, "Measuring and modeling anisotropic reflection," Comput. Graph. 26 (SIGGRAPH92), $265-272,1992$.

14. K. F. Karner, H. Mayer, and M. Gervautz, "Am image based measurement system for anisotropic reflection," Computer Graphics Forum (Eurographics '96 Proceedings) 15, 119-128 (1996).

15. R. J. Castonguay, "New generation high-speed high-resolution hemispherical scatterometer," in Optical Scattering: Application, Measurement, and Theory II, J. C. Stover, ed., Proc. SPIE 1995, 152-165 (1993).

16. K. Dana, S. Nayar, B. Ginneken, and J. J. Koenderink, "Reflectance and texture of real-world surfaces," $A C M$ Trans. Graph. 18, 1-34 (1999).

17. S. R. Marschner, S. H. Westin, E. P. F. Lafortune, and K. E. Torrance, "Image-based bidirectional reflectance distribution function measurement", Appl. Opt. 39, 2592-2600, 2000.

18. R. A. Hall, “Comparing Spectral Color Computation Methods," IEEE Comput. Graph. Appl. 19, No.4, 36-45, (1999).

19. American Society for Testing and Materials, Standard Practice for Angle Resolved Optical Scatter Measurements on Specular or Diffuse Surfaces (American Society for Testing and Materials, West Conshohocken, PA, 1996), Standard E 1392-96.

20. S. C. Foo, "A gonioreflectometer for measuring the bidirectional reflectance of material for use in illumination computation,” M.S. Thesis (Cornell University, Ithaca, NY, 1997).

21. H. Li and K. E. Torrance, "Validation of the Gonioreflectometer", Technical Report PCG-03-2 (Program of Computer Graphics, Cornell University, Ithaca, NY, 2003).

22. A. Grynberg and G. Ward, "A new tool for reflectometry," Monograph 161, (Lawrence Berkeley National Laboratory, Berkeley, CA, 1990).

23. P. Shirley and K. Chiu, "Notes on adaptive quadrature on the hemisphere," Technical Report 441 (Department of Computer Science, Indiana University, Bloomington, IN, 1994).

24. A. W. Springsteen, J. Leland and T. M. Ricker, A guide to reflectance materials and coatings, Labsphere Corporation (North Sutton, NH, 1990). 
25. K. E. Torrance and E. M. Sparrow, "Theory for off-specular reflection from roughened surfaces," J. Opt. Soc. Am. 57, 1105-1114 (1967).

26. E. P. F. Lafortune, S. C. Foo, K. E. Torrance, and D. P. Greenberg, "Non-linear approximation of reflectance functions," Comput. Graph. Proc., Annual Conference Series (SIGGRAPH97), 117-126 (1997).

27. L. Gritz and J. K. Hahn, "BMRT: A global illumination implementation of RenderMan standard," Journal of Graphics Tools 1, No.3, 29-47 (1996).

Table 1: Technical Parameters of the Gonioreflectometer

\begin{tabular}{|c|c|}
\hline Light Source: & $\begin{array}{l}\text { Source Power Drift: } 0.03 \%+5 \mathrm{~mA} \\
\text { Solid Angle: } 2.4 \times 10^{-5} \mathrm{sr}\end{array}$ \\
\hline Detector: & $\begin{array}{l}\text { Solid Angle: } 0.00128 \mathrm{sr} \\
\text { Signal Readout Resolution: } 16 \text { bit } \\
\text { Detector Dynamic Range: } 1: 22,000 \\
\text { Electron Sensitivity: } 1,900 \text { photoelectrons/count } \\
\text { Spectral Range: } 386 \mathrm{~nm}-711 \mathrm{~nm} \\
\text { Spectral Resolution: } 2.8 \mathrm{~nm}\end{array}$ \\
\hline Mechanical System: & $\begin{array}{l}\text { Rotation Stage } 1 \text { Range: } 0^{\circ} \sim 360^{\circ} \\
\text { Rotation Stage } 1 \text { Resolution: } 0.01^{\circ} \\
\text { Rotation Stage } 2 \text { Range: } 0^{\circ} \sim 180^{\circ} \\
\text { Rotation Stage } 2 \text { Resolution: } 0.01^{\circ} \\
\text { Rotation Stage } 3 \text { Range: } 7^{\circ} \sim 180^{\circ} \\
\text { Rotation Stage } 3 \text { Resolution: } 0.013^{\circ} \\
\end{array}$ \\
\hline Test Sample: & $\begin{array}{l}\text { Dimension of Sample Surface: } 130 \mathrm{~mm} \times 130 \mathrm{~mm} \\
\text { Illumination Spot: } 25 \mathrm{~mm} \text { diameter } \\
\text { Uniformity of Illumination: within } \pm 5 \% \\
\text { Maximum Angle of Reflection: } \geq 85^{\circ}\end{array}$ \\
\hline
\end{tabular}

Table 2 Reciprocity assessment on Spectralon with the Gonioreflectometer

\begin{tabular}{|r|r|l|l|}
\multicolumn{1}{c}{$\theta_{i}$} & $\theta_{r}$ & \multicolumn{1}{l}{ Signal Reading } & \multicolumn{1}{l|}{ Relative Error } \\
\hline 40 & 80 & 1369 & $0.58 \%$ \\
80 & 40 & 1361 & $0.60 \%$ \\
\hline 46 & 74 & 1497 & \\
74 & 46 & 1488 & $0.64 \%$ \\
\hline 50 & 70 & 1555 & $0.19 \%$ \\
70 & 50 & 1545 & \\
\hline 54 & 66 & 1582 & \\
66 & 54 & 1579 & \\
\hline
\end{tabular}

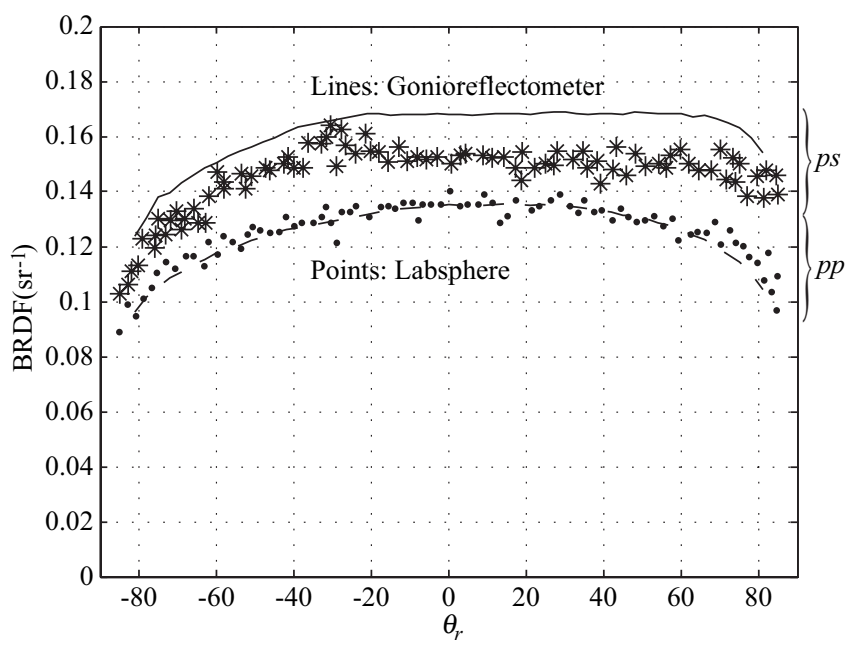

Figure 4. Comparison of measured and reference BRDFs for Spectralon in the plane of incidence for $\theta_{i}=20^{\circ}, \lambda=633 \mathrm{~nm}$, and ps and pp components of polarization 


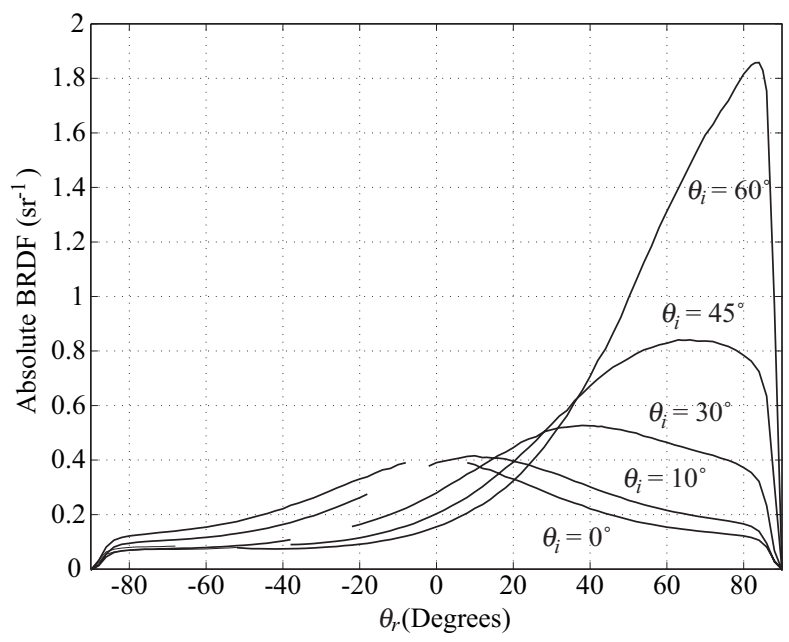

Figure 5. Incidence-plane BRDF of rough aluminum surface for several incidence angles $\theta_{i} ; \lambda=550 \mathrm{~nm}$

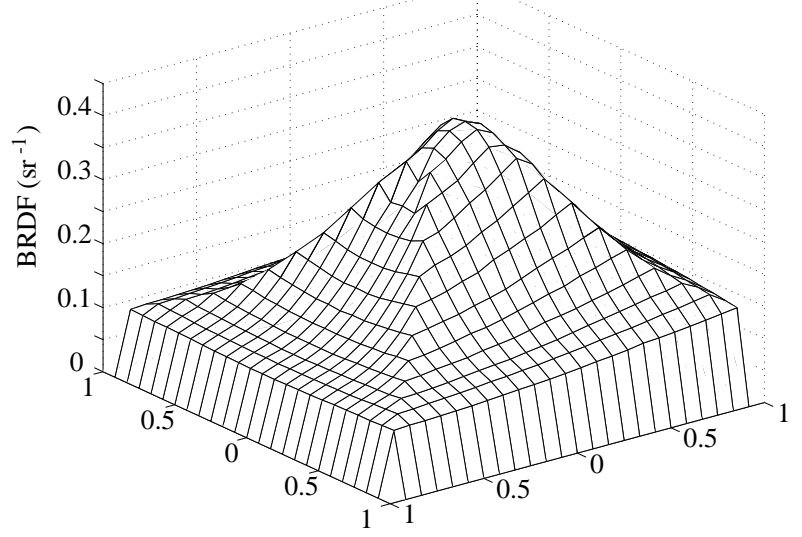

Figure 6. BRDF of rough aluminum over the mapped reflection hemisphere for $\theta_{i}=10^{\circ}$ and $\lambda=550 \mathrm{~nm}$

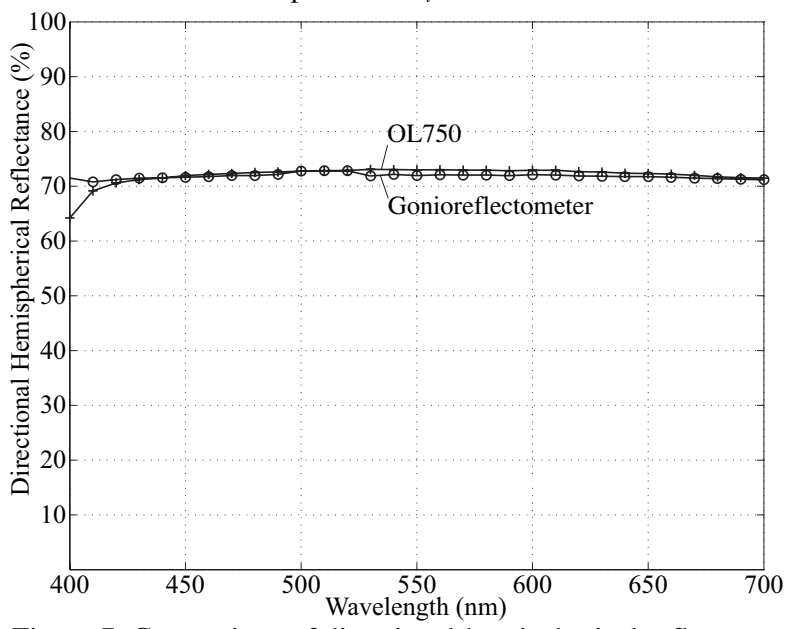

Figure 7. Comparison of directional-hemispherical reflectance of rough aluminum as measured by two instruments, $\theta_{i}=10^{\circ}$

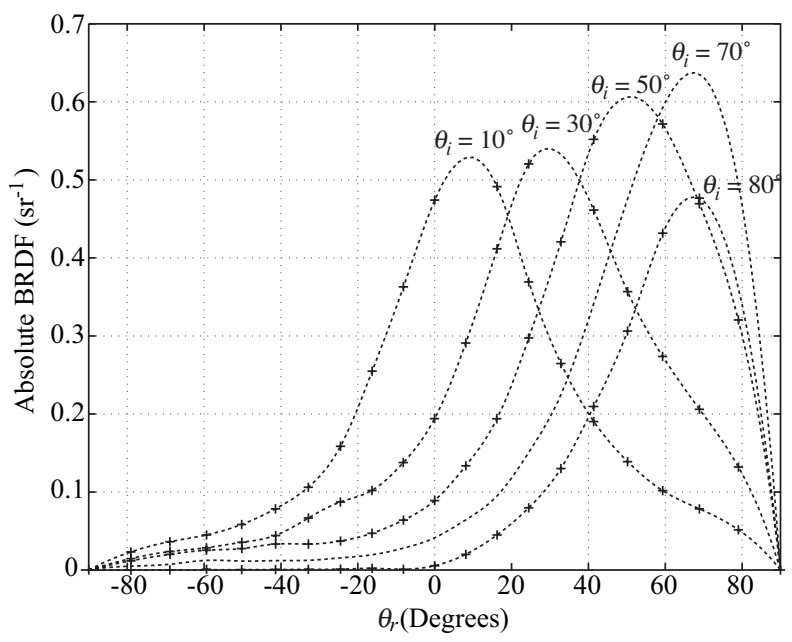

Figure 8. Incidence-plane BRDF of metallic silver paint for several incidence angles $\theta_{i} ; \lambda=550 \mathrm{~nm}$

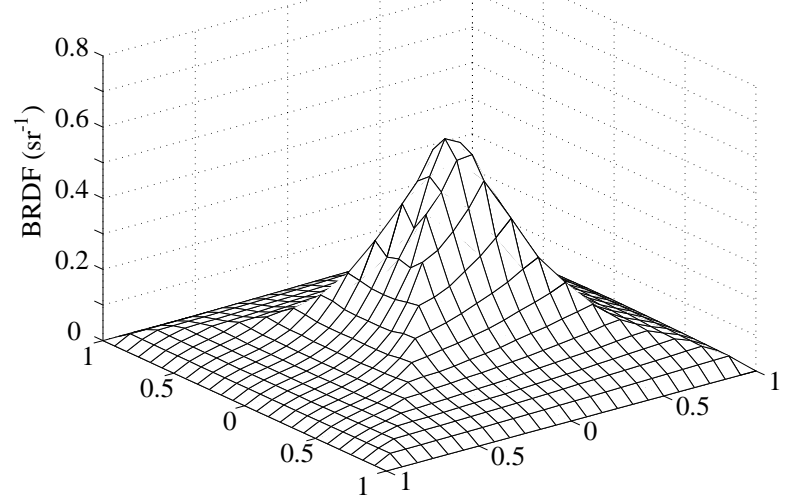

Figure 9. BRDF of metallic silver paint over the mapped reflection hemisphere for $\theta_{i}=10^{\circ}$ and $\lambda=550 \mathrm{~nm}$

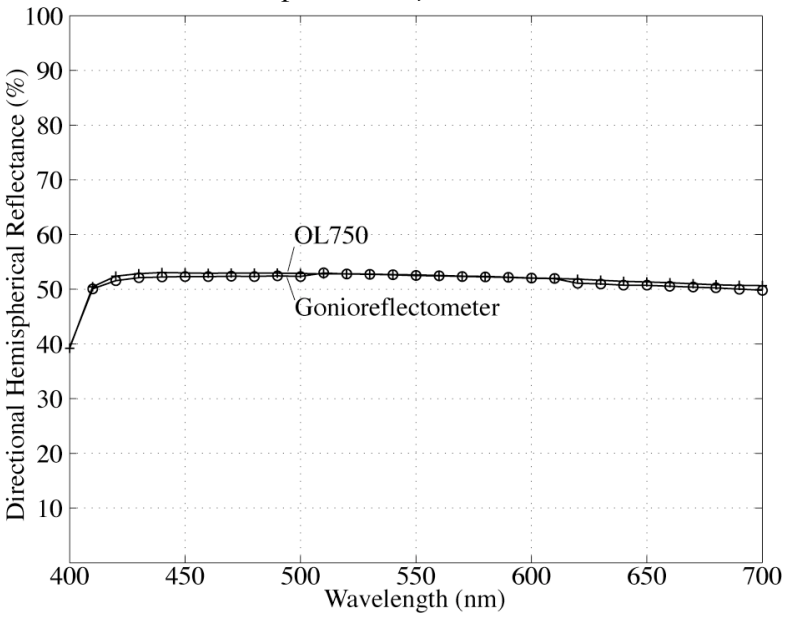

Figure 10. Comparison of directional-hemispherical reflectance of metallic silver paint as measured by two instruments, $\theta_{i}=1$ 


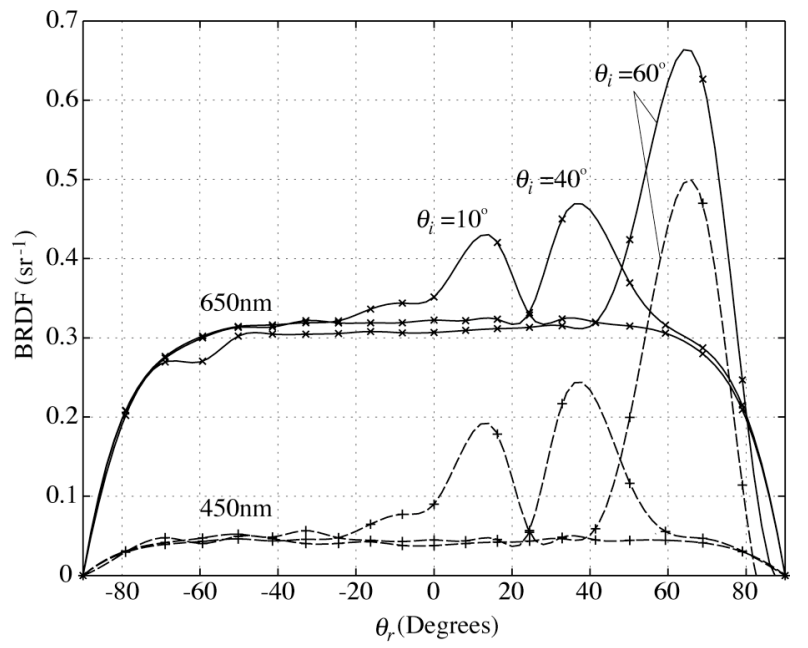

Figure 11. Incidence-plane BRDF of glossy yellow paint for several incidence angles $\theta_{i} ; \lambda=450,650 \mathrm{~nm}$

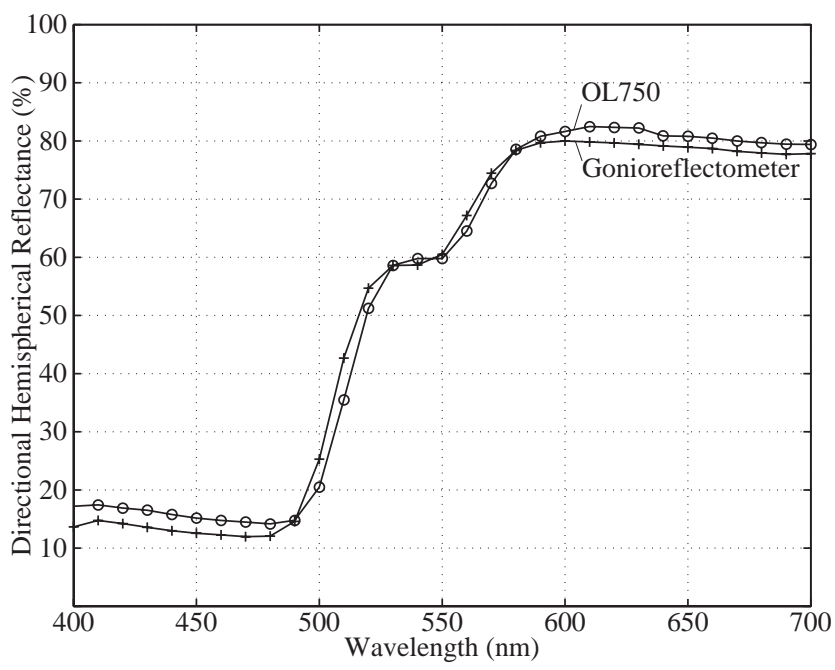

Figure 12. Comparison of directional-hemispherical reflectance of glossy yellow paint as measured by two instruments, $\theta_{i}=10^{\circ}$

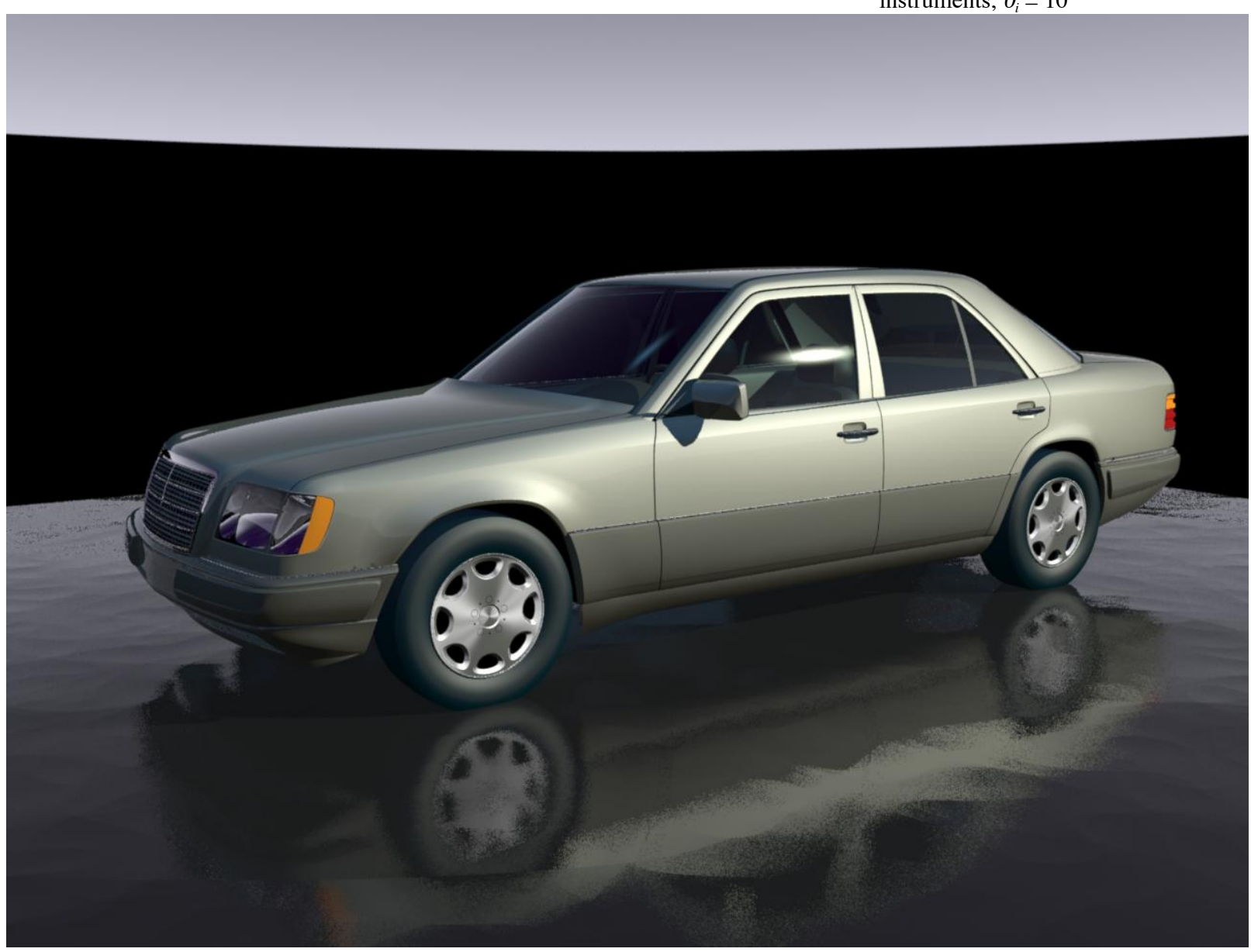

Figure 13. Computer-generated image based on measured BRDF of the metallic silver paint 